\title{
Processos metodológicos para o estudo da caminhabilidade em Cachoeira do Sul mediante a técnica Best-Worst Scaling
}

\author{
Methodological procedures for studying walkability in Cachoeira do Sul throught Best-Worst
} Scaling technique

\author{
Letícia Oestreich ${ }^{1}$, Jean Augusto Lemes ${ }^{2}$, Tânia Batistela Torres ${ }^{3}$, Brenda Medeiros Pereira ${ }^{4}$ e \\ Alejandro Ruiz-Padillo 5 \\ 1,2,3,4 Laboratório de Mobilidade e Logística, Universidade Federal de Santa Maria, Campus de Cachoeira do Sul, \\ Brasil \\ leticia.oestreich@hotmail.com; jean.1.augusto@hotmail.com; taniabatistela@gmail.com; brenda.pereira@ufsm.br \\ ${ }^{5}$ Laboratório de Mobilidade e Logística, Universidade Federal de Santa Maria, Campus de Cachoeira do Sul \\ Laboratório de Sistemas de Transportes, Universidade Federal do Rio Grande do Sul, Porto Alegre, Brasil \\ alejandro.ruiz-padillo@ufsm.br
}

\begin{abstract}
Resumo
As cidades enfrentam cada vez mais problemas na mobilidade urbana, estudos sobre a influência dos transportes na promoção da qualidade de vida servem como importantes instrumentos para tomadores de decisão para o planejamento urbano. Neste contexto, pesquisas para a identificação de características que tornam uma cidade mais caminhável são ferramentas auxiliares na projeção de ambientes mais sustentáveis, seguros, saudáveis, acessiveis e agradáveis. Este artigo tem por objetivo apresentar os processos metodológicos utilizados para a elaboração do instrumento de pesquisa necessário para a futura aplicação da técnica best-worst scaling que, no estudo da caminhabilidade (em inglês walkability), permite a determinação das características mais importantes para a promoção da caminhada no caso de uma cidade brasileira, interiorizada, de porte médio. Entre as etapas metodológicas apresentadas se encontram: os métodos de distribuição geoespacial e caracterização dos bairros da cidade de estudo, estratificação da amostra e cálculo amostral, variáveis consideradas para análise, coleta de dados e estruturação do questionário de caminhabilidade.
\end{abstract}

Palavras-chave: Metodologia; Caminahbilidade; Best-Worst Scaling

\section{Abstract}

Cities increasingly face problems in urban mobility. Studies on the influence of transport to promote quality of life serve as important tools for decision-making regaring urban planning. In this context, studies to identify the characteristics to design a walkable city are supporting tools in building more sustainable, safer, healtier, more accessible and livable urban environment. This paper aims to present the methodological procedures taken to elaborate the necessary instruments to the future application of the Best Worst Scaling technique, which in the walkablity study allows the determination of the most important characteristics to promote walking as transport. Among the methodological steps presented in this paper are the geospatial distribution methods and neighborhoods characterization in Cachoeira do Sul, a mid-sized city in Brazilian, sample stratification and calculation, variables included in the analysis, data collection, design and application of the walkability surveys 


\section{Introdução}

A promoção de mobilidade urbana sustentável e inclusiva tem sido um desafio para as cidades. A disputa por espaços urbanos, congestionamentos, acidentes viários, poluição atmosférica e sonora são externalidades percebidas de diferentes formas pelas pessoas que necessitam de deslocamento para realizar suas atividades. Por conta disso, os países membros da Organização das Nações Unidas (ONU) se comprometeram, até o ano de 2030, a adotarem medidas para proporcionar o acesso a sistemas de transportes seguros, acessíveis e sustentáveis a todas as pessoas, como parte dos Objetivos do Desenvolvimento Sustentável (do inglês, Sustainable Development Goals) (United Nations General Assembly, 2015).

Promover ambientes favoráveis ao uso de modos de transportes não motorizados e coletivos reduz o uso significativo de modos motorizados (CERVERO e DUNCAN, 2003). Por consequência, ocorre um aumento da qualidade de vida das pessoas, associado à redução das taxas de emissões e à promoção da acessibilidade para todos (NEWMAN e KENWORTHY, 1999). Além disso, cidades que promovem viagens a pé ou de bicicleta reduzem a exposição ao risco e aumentam a percepção de segurança viária devido às velocidades veiculares mais baixas, que, por consequência, diminuem as taxas de mortalidade por acidentes de trânsito (ELVIK et al., 2004; JENSEN, 2008).

Para incentivar o uso desses modos é necessário prover medidas que constituam um ambiente oportuno para o uso de transporte coletivo e não motorizado (BANISTER, 2008), uma vez que a percepção do indivíduo sobre o ambiente direcionado a esses modos vai influenciar na sua escolha por assim realizar a viagem (EWING e HANDY, 2009). Autores afirmam que as pessoas se deslocam a pé preferencialmente em locais que apresentam características como boas condições do desenho urbano (EWING e CERVERO, 2001), bairros esteticamente agradáveis (RHODES et al., 2006), calçadas largas e com boa iluminação (KIM et al., 2014), áreas recreativas (STRADLING et al., 2007), ambientes comerciais (CERVERO, 1996), boa segurança pública (KELLY et al., 2011) e boa segurança viária (DUMBAUGH e ERA, 2009).

Segundo um estudo realizado por Kim et al. (2014), ambientes que promovem viagens a pé apresentam características relacionadas a calçadas largas, cruzamentos seguros, áreas verdes e de boa iluminação. Para Ewing e Cervero (2001), uma calçada em boas condições eleva a acessibilidade para pedestres, assim como, de maneira inversa, um tráfego intenso inibe essa acessibilidade. Já a topografia do terreno acidentada pode influenciar negativamente, enquanto que áreas mais planas promovem boas percepções de caminhada entre os moradores (LESLIE et al., 2005; FERRER e RUIZ, 2016).

Para Cervero (1996) a presença de comércios e ou serviços no entorno é um estímulo para viagens a pé, preferencialmente a uma distância de 100 metros. O estudo de Stradling et al. (2007) também indicou que instalações comerciais promovem a caminhada e que a largura das calçadas, qualidade do ar, tempos de deslocamentos em travessias e segurança viária influenciam na escolha por essas viagens.

Por outro lado, possuir um veículo motorizado é um fator que desestimula a caminhada (CERVERO e DUNCAN, 2003), assim como o número de familiares empregados no domićlio aumenta a probabilidade do uso de modos motorizados, visto que as atividades fora do trabalho se conectam à rotina do uso do automóvel (GREENWALD e BOARNET, 2001). Entretanto, à medida que as distancias de deslocamentos diminuem há um aumento no número de viagens a pé (BALSAS, 2003; EWING e CERVERO, 2001).

Portanto, percebe-se uma variedade de elementos que estimulam a caminhada e que estão associados à forma urbana, percepção e comportamento dos usuários, e ambiente construído. Nesse contexto, surge o estudo da caminhabilidade (do inglês, walkability), que permite identificar quais os fatores que interferem ou estimulam a caminhada em uma cidade. Segundo Bradshaw (1993) pode ser definido por quatro características básicas (i) ambiente físico direcionado ao pedestre, (ii) densidade de comércios e serviços, (iii) ambiente influenciado por altas ou baixas temperaturas e (iv) cultura local. 
Estudar a caminhabilidade permite proporcionar de forma física as motivações que possam levar ao fomento de viagens a pé de forma natural, através da determinação dos fatores que caracterizam a qualidade do ambiente (GHIDINI, 2011). Além disso, as cidades de médio porte são as que apresentaram um maior crescimento nos últimos anos (IBGE, 2012), evidenciando a importância de um planejamento voltado a sustentabilidade para que os problemas de mobilidade não sejam agravados e as cidades tenham um crescimento saudável (JABAREEN, 2006). Para Kim et al., (2014), a prática de caminhar pode ser encorajada à medida que se melhora a qualidade do ambiente construído.

O planejamento, quando em fase de estudo, deve levar em consideração as pessoas idosas, adaptando-se a estrutura necessária para que elas caminhem com segurança e comodidade (LEE e DEAN, 2018). Essa estrutura, se bem executada ou não, influencia diretamente e indiretamente na qualidade do ambiente de caminhada, visto que as percepções dos indivíduos estão conectadas às características físicas do local (EWING e HANDY, 2009).

Dessa forma, analisar os fatores que influenciam e estimulam a caminhada pode auxiliar na formulação de medidas importantes no contexto de modelo de planejamento de cidades sustentáveis. Com base nisso, este estudo fundamenta-se em um objetivo global, que busca identificar as variáveis que influenciam na escolha por modos ativos para o caso de uma cidade interiorizada, brasileira e de médio porte. Como parte desse projeto, o objetivo deste estudo é apresentar os processos metodológicos utilizados para a elaboração do instrumento de pesquisa que servirá como base para o estudo posterior da identificação das características que tornam os bairros mais caminháveis na cidade de Cachoeira do Sul através da aplicação da técnica proposta.

A elaboração desses estudos preliminares são etapas importantes do planejamento dos transportes, pois permitem a difusão das características apontadas pelos entrevistados e as variáveis observadas no bairro de residência, a fim de identificar o comportamento dos usuários diante o ambiente em que ele está inserido. A aplicação dessa pesquisa foi baseada no estudo de Larrañaga et al. (2016), no qual é proposto o uso da técnica Best-Worst Scaling que irá avaliar o impacto das características na escolha de viagens a pé, a fim de definir quais variáveis são indispensáveis para o planejamento sustentável da cidade.

\section{Metedologia}

A metodologia utilizada neste trabalho é composta pela coleta de dados e amostragem, pelo o modelo probabilístico Best-Worst Scaling e a elaboração do questionário com o propósito de identificar os fatores que afetam a caminhabilidade na cidade. O estudo foi aplicado no caso do município brasileiro de Cachoeira do Sul, a cidade possui uma população em torno de 85 mil habitantes (IBGE, 2012), localizada no interior do Estado do Rio Grande do Sul a $200 \mathrm{~km}$ da capital. A cidade possui uma, estimada considerando apenas a área urbana.

\subsection{Coleta de dados e amostragem}

Como forma de captar as informações de maneira a obter um nível de confiabilidade aceitável, o estudo dedicou-se a coletar informações que pudessem descrever a população em estudo segundo suas características pessoais e segundo as características do bairro de residência. Para tanto, a estratificação da amostra para a unidade espacial de bairro foi realizada a partir de variáveis que pudessem influenciar na caminhada e caracterizassem os bairros da cidade. Os bairros da cidade foram caracterizados segundo três variáveis: (i) rendimento per capita; (ii) declividade média e (iii) densidade de comércios e serviços. Os entrevistados foram caracterizados conforme a idade e o sexo. A seleção da amostra é dada através do cálculo estatístico apresentado na equação 1, descrito por (SPIEGEL, 2009). 


$$
n=\frac{N \times Z^{2} \times p \times(1-p)}{Z^{2} p \times(1-p)+e^{2} \times(N-1)}
$$

onde $n$ é o tamanho da amostra, $N$ é a população em estudo, $Z$ é a variável padrão associada ao nível de confiança, $p$ é a verdadeira probabilidade da ocorrência do evento e $e$ é o erro amostral.

\subsection{Modelo probabilístico Best-Worst Scaling}

A técnica Best-Worst Scaling (B/W) é uma abordagem de escolha discreta baseada nos princípios da utilidade aleatória em um contexto de comparação par a par (LARRAÑAGA et al., 2016). O método foi proposto por Finn e Louviere (1992) e sua funcionalidade é fundamentada na ideia de que são apresentados um conjunto de atributos e o entrevistado escolhe o melhor (best) e o pior (worst) dentro das opções disponíveis de forma a se obter a importância de cada atributo. Na prática, é apresentado ao indivíduo um par de cenários com as características propostas, os cenários se diferenciam pelo nível de cada atributo. O entrevistado escolhe o cenário que considera mais caminhável, dentro desse cenário, ele indica qual melhor e a pior característica.

Para o caso estudado, estruturou-se um questionário com um conjunto de nove variáveis que influenciam a escolha por viagens a, pé baseados nas características propostas por Larrañaga et al. (2016). O questionário foi organizado em duas fases de forma que a apresentação das variáveis foi diluída para captar melhor as informações, dentre as características a serem avaliadas incluem: presença de comércios e serviços, risco de acidentes de tráfego, número de policiais por habitantes, atratividade do bairro, declividade, largura útil da calçada, condições do pavimento e conectividade viária.

\section{Resultados e discussões}

Esta seção apresenta os processos decorrentes da metodologia utilizada para preparação da pesquisa e elaboração do instrumento utilizado para coleta dos dados necessários para a análise da caminhabilidade em Cachoeira do Sul. Portanto, tratase de resultados parciais do estudo maior que servirão como base para a aplicação da técnica que irá identificar as características que tornam os bairros mais caminháveis. Esses resultados metodológicos incluem a distribuição e classificação dos bairros da cidade de Cachoeira do Sul, bem como a escolha da amostra populacional estratificada que deverá ser atingida na aplicação dos questionários posteriormente.

\subsection{Distribuição e classificação dos bairros da cidade}

Em um primeiro momento, o estudo se dedicou a obter um georreferenciamento dos bairros da cidade. Para tanto, a partir de informações geoespaciais dos setores censitários da cidade realizou-se, com o auxílio de ferramenta GIS, o cálculo do centroide dos setores, e com base nesses dados identificou-se a estimativa da localização geográfica do bairro em que se encontrava cada setor censitário. Foi identificado um total de 28 bairros para a cidade de Cachoeira do Sul, que podem ser visualizados na Figura 1.

Para a análise e classificação dos bairros da cidade com base em características que podem influenciar na caminhabilidade, utilizou-se como parâmetros da estratificação dos setores censitários três variáveis: (i) rendimento per capita (IBGE, 2012); (ii) declividade média absoluta obtida por Modelo Digital de Superfície (INPE, 2008); e a (iii) densidade de comércios e serviços que foi obtida através da geocodificação dos dados da Prefeitura da cidade de Cachoeira do Sul, fornecidos pela Secretaria 
Municipal de Indústria e Comércio de Cachoeira do Sul (SMIC). Todos os dados foram coletados e analisados através de uma ferramenta GIS. Na Figura 1 é apresentada a distribuição geoespacial dos dados de comércios e serviços na área urbana da cidade.

Figura 1 - Distribuição geoespacial dos comércios e serviços na área urbana da cidade de estudo

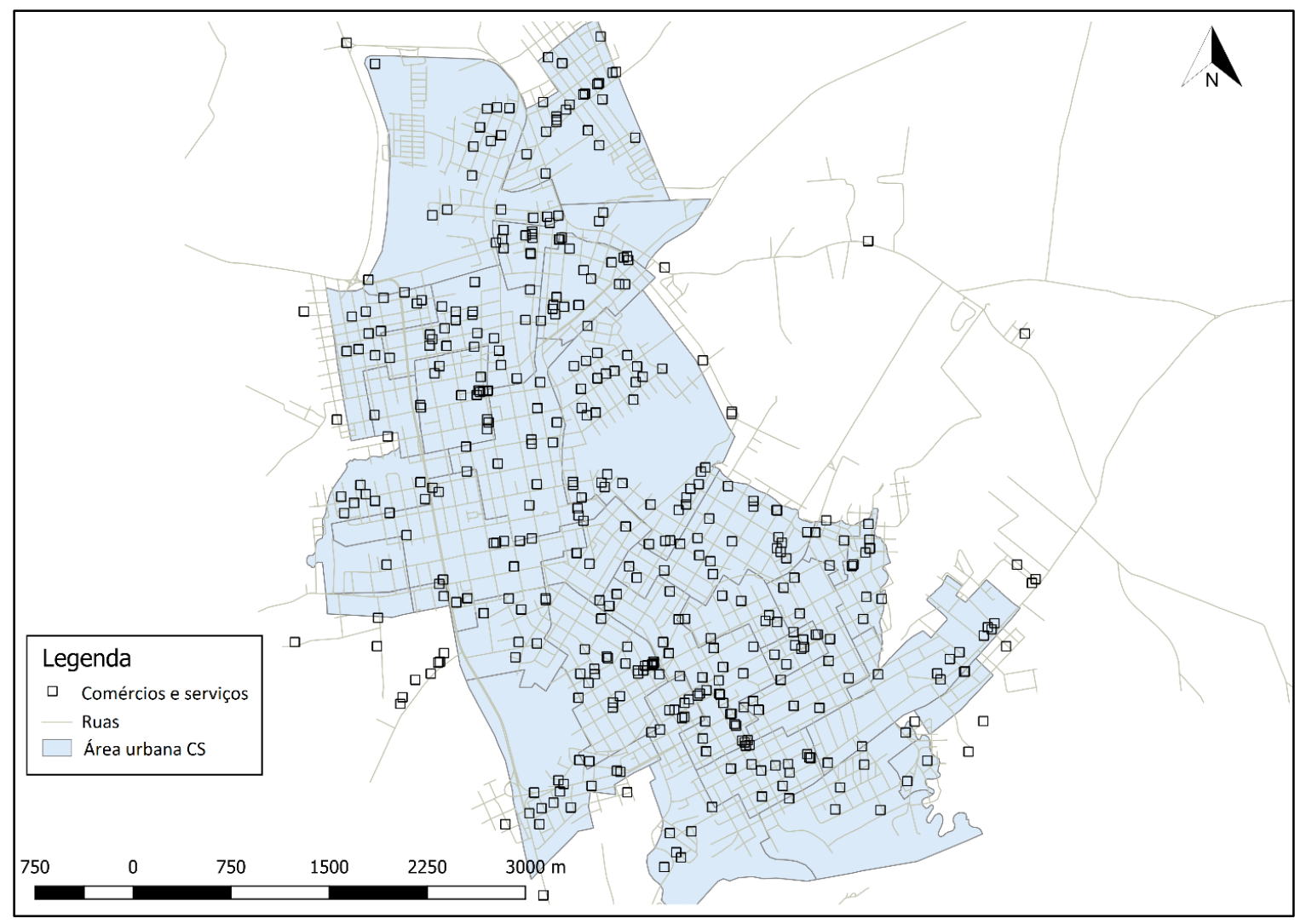

Como forma de classificar os bairros entre essas características em estudo, realizou-se, com o auxílio de distribuição estatística, a análise qualitativa para a definição dos níveis das variáveis segundo a conveniência do trabalho. Dada essa análise, obteve-se para as variáveis Rendimento Per capita, Declividade Média, Densidade de Comércios e Serviços os níveis e seus respectivos intervalos para as variáveis de estudo são apresentados na Tabela 1.

Tabela 1 - Variáveis de análise e seus respectivos níveis de classificação

\begin{tabular}{ll}
\hline \multicolumn{1}{c}{ Variável de análise } & \multicolumn{1}{c}{ Níveis } \\
\hline Rendimento per-capita & $-1)$ Baixo: $>\mathrm{R} \$ 1000$ \\
\hline Declividade média & -2 ) Alto: $<\mathrm{R} \$ 1000$ \\
& $-1)$ Baixa: $<7 \%$ \\
\hline Densidade de comércios e serviços & $-2)$ Alta: $>7 \%$ \\
\hline
\end{tabular}


Foram consideradas três variáveis de estratificação, com seus respectivos níveis (2, 2 e 3). Sendo assim, obtém-se 12 possíveis combinações (12 estratos) entre as variáveis e seus níveis, que são apresentadas na Tabela 2.

Tabela 2 - Combinação das variáveis dos estratos em estudo

\begin{tabular}{cccc}
\hline Estrato & Rendimento per-capita & Declividade média & Densidade de comércios \\
\hline $\mathbf{1}$ & 1 & 1 & 1 \\
$\mathbf{2}$ & 1 & 1 & 2 \\
$\mathbf{3}$ & 1 & 1 & 3 \\
$\mathbf{4}$ & 1 & 2 & 1 \\
$\mathbf{5}$ & 1 & 2 & 2 \\
$\mathbf{6}$ & 1 & 2 & 3 \\
$\mathbf{7}$ & 2 & 1 & 1 \\
$\mathbf{8}$ & 2 & 1 & 2 \\
$\mathbf{9}$ & 2 & 1 & 3 \\
$\mathbf{1 0}$ & 2 & 2 & 1 \\
$\mathbf{1 1}$ & 2 & 2 & 2 \\
$\mathbf{1 2}$ & 2 & 2 & 3 \\
\hline
\end{tabular}

Como o interesse da aplicação do estudo é nos bairros da cidade e os dados foram obtidos por setores censitários, foi necessário agregar as informações a nível de bairro. O agrupamento das variáveis para a obtenção do estrato foi realizado através da ponderação das características por área dos setores censitários pertencentes a cada bairro. Como resultado, todos os bairros ficaram classificados em apenas 5 dos estratos da análise, como é possível perceber na Figura 2.

Figura 2 - Dados geoespaciais dos bairros da cidade em estudo e o estrato pertencente

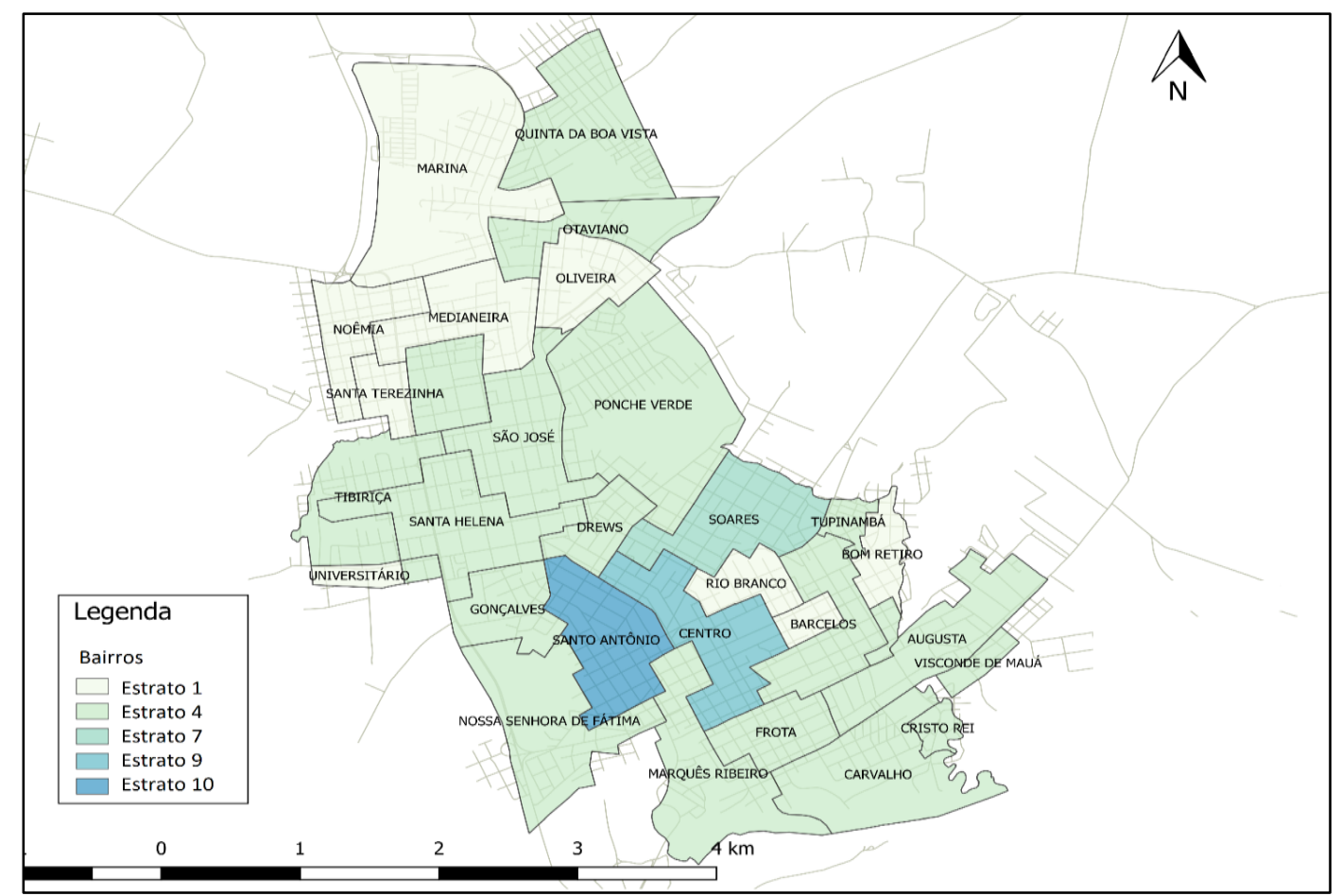


Com relação aos estratos aos quais cada bairro da cidade pertence (Figura 2), observa-se que em sua maioria os bairros são caracterizados pelo estrato 4 , ou seja, bairros que possuem um baixo rendimento per-capita, alta declividade e baixa densidade de comércios e serviços. O bairro denominado centro foi o único que pertence ao estrato 9 , caracterizado por um auto rendimento per-capita, baixa declividade e alta densidade de comércios e serviços. Esses dados demonstram a consistência do estudo, visto que as regiões centrais das cidades possuem uma maior concentração dos serviços e comércios da cidade, além de que a baixa declividade nessas regiões também permite essa configuração.

\subsection{Estratificação da amostra populacional}

Como forma de obter uma amostra representativa da população, foi estimada a amostra necessária para o estudo e aplicação dos questionários. A partir dos dados da população residente em Cachoeira do Sul - 83.827 habitantes, conforme IBGE (2012) - a amostra foi calculada, a partir da equação 1, considerando um nível de confiança de $90 \%$, erro admissível de $5 \%$ e um coeficiente de variação de $20 \%$.

Como resultado do cálculo amostral, foi obtido que para obter-se uma representatividade adequada da população deverá ser aplicado um mínimo de 180 questionários; como foram atingidos cinco estratos para a cidade, é necessário que seja alcançado um mínimo de 35 questionários para cada estrato. Adicionalmente, a amostra populacional deve ser representativa segundo a subdivisão entre sexo e idade. Na Tabela 3 é apresentado o resultado do número de questionários para cada estrato segundo a representatividade da população com base no censo do IBGE (2012).

Tabela 3 - Amostra representativa necessária para cada estrato do estudo

\begin{tabular}{lcccc}
\hline & Soma de 15 a 29 anos & Soma de 30 a 59 anos & Soma de mais de 60 & Soma de Total \\
\hline Feminino & 5 & 9 & 4 & 18 \\
Masculino & 5 & 9 & 3 & 17 \\
Total Geral & 10 & 18 & 7 & 35 \\
\hline
\end{tabular}

\subsection{Aplicação dos questionários e processamento dos dados}

Selecionados os bairros de estudo e as amostras populacionais a serem atingidas para cada estrato, a coleta de dados foi realizada de duas formas: (i) questionário online: disponibilizado para toda a comunidade e divulgado por meio de recursos eletrônicos; (ii) entrevistas nos domicílios: coleta das respostas através da alocação de entrevistadores nos bairros para realizar entrevistas domiciliares. A primeira técnica facilitou a obtenção da maior parte dos questionários necessários de forma aleatória, mas foi preciso utilizar a segunda forma de coleta para completar a amostra definida de acordo com a estratificação, assim como sua distribuição correta nos bairros selecionados.

Uma vez obtidos todos os questionários, os dados coletados foram processados para facilitar seu tratamento mediante a técnica Best-Worst Scaling com apoio de recursos informáticos, como era o objetivo do trabalho. 


\section{Conclusões}

Diante ao cenário atual, onde os problemas de mobilidade urbana têm afetado o cotidiano das pessoas, é preciso que sejam tomadas medidas para melhorar as condições dos deslocamentos. Nesse contexto, a engenharia pode promover o uso de modos de transportes mais sustentáveis através de ações que criam ambientes favoráveis ao uso desses modos. Assim, os estudos para avaliar as características que tornam os bairros de uma cidade mais caminháveis são importantes instrumentos para a tomada de decisão e planejamento das cidades voltado à sustentabilidade e inclusão de todos os usuários.

A realização desses estudos requer um desempenho inicial relacionado à coleta de dados para o levantamento do problema, caracterização das cidades e aplicação dos questionários para identificação das variáveis que influenciam a caminhada. Por conta disso, é visível a importância da formulação de bancos de dados atualizados que contenham características das cidades, bem como padrões de deslocamentos e características que afetam a qualidade das viagens para que o planejamento dos transportes seja facilitado e estudos semelhantes a esses possam ser valorizados e utilizados como importantes instrumentos para a tomada de decisão.

Assim, os resultados parciais apresentados neste trabalho, incluem os procedimentos metodológicos realizados para a coleta de dados necessários para aplicação da técnica Best-Worst Scaling. As etapas resultantes deste estudo servirão como auxiliares na análise do impacto das características do entorno na escolha dos cidadãos por viagens a pé e, além disso, irão contribuir para a compreensão das preferências para a caminhada dos entrevistados. A técnica foi adaptada para uma cidade de Cachoeira do Sul, caracterizada como uma cidade de médio porte, localizada no interior do Estado do Rio Grande do Sul (Brasil).

Espera-se com a finalização deste projeto de estudo indicar quais são os atributos (variáveis) mais importantes e que afetam a caminhabilidade nos bairros da cidade, auxiliando no diagnóstico para planejamento de medidas públicas que melhorem as condições de mobilidade do pedestre nos locais específicos. Além disso, a identificação desses fatores associados às características da cidade podem gerar modelos de preferências para outras cidades com características semelhantes, contribuindo para a formulação de medidas padrões para tornar as cidades mais caminháveis e consequentemente mais sustentáveis.

\section{Referências}

BALSAS C J L. Sustainable transportation planning on college campuses. Transport Policy. 2003;10(1):35-49.

BANISTER D. The sustainable mobility paradigm. Transport Policy. 2008;15(2):73-80.

BRADSHAW C. Creating - and Using - a Rating System for Neighbourhood Walkability. Hearth Healt. Paper presented at the 14th International Pedestrian Conference, Boulder, CO. 1993. Available from: http://hearthhealth.wor dpress.com/about/previously-published-works/feet-first-early/creaing-and-using-a-rating-system-for-neighbourhoodwalkability-towards-an-agenda-for-local-heroes-1993

CERVERO R, DUNCAN M. Walking, Bicycling, and Urban Landscapes: Evidence from the San Francisco Bay Area. American Journal of Public Health. 2003;93(9):1478-1483.

CERVERO R. Mixed land-uses and commuting: Evidence from the American Housing Survey. Transportation Research Part A: Policy and Practice. 1996;30(5):361-377.

DUMBAUGH E, RAE, R. Safe urban form: revisiting the relationship between community design and traffic safety. Journal of the American Planning Association. 2009;75(3):309-329.

ELVIK R, HOYE A, VAA T, SORENSEN M. The handbook of road safety measures; 2004 
EWING R, CERVERO R. Travel and the Built Environment: A Synthesis. Transportation Research Record: Journal of the Transportation Research Board. 2001;1780(1):87-114.

EWING R, HANDY S. Measuring the unmeasurable: Urban design qualities related to walkability. Journal of Urban Design. 2009;14(1):65-84.

FERRER S, RUIZ T. The impact of the built environment on the decision to walk for short trips: Evidence from two Spanish cities. Transport Policy. 2016;67:111-120.

FINN A, LOUVIERE J J. Determining the appropriate response to evidence of public concern: The case of food safety. Journal of Public Policy and Marketing. 1992;11(1):12-25.

GHIDINI R. A caminhabilidade: medida urbana sustentável. Revista dos Transportes Públicos - ANTP. 2011,33(1):23-33.

GREENWALD M, BOARNET M. Built Environment as Determinant of Walking Behavior: Analyzing Nonwork Pedestrian Travel in Portland, Oregon. Transportation Research Record: Journal of the Transportation Research Board. 2001;1780:33-41.

IBGE. Perfil dos Municípios Brasileiros 2012. Instituto Brasileiro de Geografia e Estatística. 2011. Disponível em: ftp://ftp.ibge.gov.br/Perfil_Municipios/2011/munic2011.pdf.

INPE. Topodata: Banco de Dados Geomorfológicos do Brasil. Instituto Nacional de Pesquisas Espaciais. 2008. Available from: http://www.dsr.inpe.br/topodata/acesso.php.

JABAREEN Y R. Sustainable urban forms: Their typologies, models and concepts. Journal of Planning Education and Research. 2006;26(1):38-72.

JENSEN, S U. How to obtain a healthy journey to school. Transportation Research Part A: Policy and Practice. 2008;42(3):475-486.

KELLY C E, TIGHT M R, HODGSON F C, PAGE M W. A comparison of three methods for assessing the walkability of the pedestrian environment. Journal of Transport Geography. 2011;19(6):1500-1508.

KIM S, PARK S, LEEJ S. (2014) Meso- or micro-scale? Environmental factors influencing pedestrian satisfaction. Transportation Research Part D: Transport and Environment. 2014;3:10-20.

LARRAÑAGA A M, CYBIS H B B, ARELLANA J, RIZZI L I, STRAMBI O. Estimando a importância de características do ambiente construído para estimular bairros caminháveis usando Best-Worst Scaling. Transportes, 2016;24(2):946-1958.

LESLIE E, SAELENS B, FRANK L, OWEN N, BAUMAN A, COFFEE N, HUGO, G. Residents' perceptions of walkability attributes in objectively different neighbourhoods: A pilot study. Health and Place. 2005;11(3):227-236.

NEWMAN P, KENWORTHY J R. Sustainability and Cities: Overcoming Automobile Dependence. Island Press, Ed. EUA: Washington DC; 1999.

RHODES R, BROWN S, MCINTYRE C. Integrating the perceived neighborhood environment and the theory of planned behaviour when predicting walking in a Canadian adult sample. American Journal of Health Promotion. 2006;(21):110-118.

SPIEGEL M R. Estatística. 4 ed. 597 p. São Paulo: Bookman; 2009.

STRADLING S G, ANABLE J, CARRENO M. Performance, importance and user disgruntlement: A six-step method for measuring satisfaction with travel modes. Transportation Research Part A: Policy and Practice. 2007;41(1): 98-106.

UNITED NATIONS GENERAL ASSEMBLY. Transforming our world: The 2030 agenda for sustainable development. Sustainable Development Goal; 2015. Available from: https://sustainabledevelopment.un.org/c ontent/documents/2252030\%20Agenda\%20for\%20Sustainable\%20Development $\% 20$ web.pdf. 